ORIGINAL ARTICLE

www.nature.com/leu

\title{
The Epstein-Barr virus nuclear antigen-1 promotes telomere dysfunction via induction of oxidative stress
}

\author{
SA Kamranvar and MG Masucci \\ Department of Cell and Molecular Biology, Karolinska Institutet, Stockholm, Sweden
}

\begin{abstract}
The Epstein-Barr virus (EBV) nuclear antigen (EBNA)-1 promotes the accumulation of chromosomal aberrations in malignant $B$ cells by inducing oxidative stress. Here we report that this phenotype is associated with telomere dysfunction. Stable or conditional expression of EBNA1 induced telomere abnormalities including loss or gain of telomere signals, telomere fusion and heterogeneous length of telomeres. This was accompanied by the accumulation of extrachromosomal telomeres, telomere dysfunction-induced foci (TIFs) containing phosphorylated histone $\mathrm{H} 2 \mathrm{AX}$ and the DNA damage response protein 53BP1, telomere-associated promyelocytic leukemia nuclear bodies (APBs), telomeric-sister chromatid exchanges and displacement of the shelterin protein TRF2. The induction of TIFs and APBs was inhibited by treatment with scavengers of reactive oxygen species (ROS) that also promoted the relocalization of TRF2 at telomeres. These findings highlight a novel mechanism by which EBNA1 may promote malignant transformation and tumor progression.
\end{abstract}

Leukemia (2011) 25, 1017-1025; doi:10.1038/leu.2011.35

published online 11 March 2011

Keywords: EBV; EBNA1; telomere; TIF; APB; ROS

\section{Introduction}

Genetic instability associated with telomere dysfunction is an early event in tumorigenesis. ${ }^{1,2}$ The telomeres consist of 3-12 kb long repeats of the hexameric nucleotide 5'-TTAGGG-3' bound to a protein complex that includes TRF1, TRF2, POT1, TIN2, TPP1 and RAP1, collectively known as the shelterin complex. ${ }^{3,4}$ The repeats terminate with a G-rich 100-200 nucleotide long single-strand $3^{\prime}$ overhang that is sequestered in a telomere loop, ${ }^{5}$ which prevents the recognition of chromosome termini as double-strand breaks. ${ }^{6}$ Telomeric DNA is synthesized by telomerase, an RNA-dependent reverse transcriptase complex whose activity is tightly regulated by subcellular localization and by the activity of the shelterin proteins. In particular, TRF1 and TRF2 control the replication and protection of telomeres and function as negative regulators of telomere length, ${ }^{7,8}$ whereas the poly-(ADP-ribose)-polymerase tankyrase promotes telomere elongation by regulating the binding of TRF1 to telomeric DNA. ${ }^{9}$

The telomeres are shortened at each cell division because of incomplete replication of the chromosome termini. When the length falls below a critical threshold, p53 is activated and induces a p16- and retinoblastoma protein-dependent replica-

Correspondence: Professor MG Masucci, Department of Cell and Molecular Biology, Karolinska Institutet, Box 285, Stockholm S-171 77, Sweden.

E-mail: maria.masucci@ki.se

Received 13 July 2010; revised 4 January 2011; accepted 28 January 2011; published online 11 March 2011 tive senescence. ${ }^{10,11}$ In the absence of functional checkpoints, progressive shortening and defects of telomere capping elicit DNA damage responses and repair of the chromosome ends by homologous recombination or non-homologous end joining, which promotes chromosomal aberrations and genomic instability. ${ }^{12}$ In most cancers, telomere length is maintained by upregulation of telomerase activity, ${ }^{13}$ but a telomerase-independent mechanism, referred to as alternative lengthening of telomeres (ALT), operates in some tumors. ${ }^{14-17}$ One important hallmark of ALT is the presence of heterogeneous telomeres that range from almost undetectable to $>50 \mathrm{~kb}$ in length. ${ }^{18}$ Another identifiable marker is the presence of promyelocytic leukemia nuclear bodies containing telomeric DNA and shelterin proteins known as ALT-associated promyelocytic leukemia nuclear bodies (APBs). ${ }^{16}$ In addition to promyelocytic leukemia (PML) and SP100, APBs also contain several DNA repair proteins, ${ }^{19}$ suggesting that they may constitute a DNA damage response triggered by uncapped or damaged telomeres. ${ }^{20,21}$ The association between ALT and dysfunctional telomeres is further supported by the occurrence of extrachromosomal telomere sequences, frequently t-circles, and telomeric-sister chromatid exchanges in ALT-positive cells. ${ }^{18}$

Epstein-Barr virus (EBV) has been implicated in the pathogenesis of a variety of lymphoid and epithelial cell malignancies including post-transplant lymphomas, endemic Burkitt's Lymphoma, nasopharyngeal carcinoma, a subset of Hodgkin's disease and gastric carcinoma. ${ }^{22}$ Infection of B lymphocytes is accompanied by the expression of viral genes, which sustains the establishment of EBV-carrying lymphoblastoid cell lines with extended growth potential in vitro. Their products include six EBV nuclear antigens (EBNA1, -2, -3A, -3B, -3C and -LP), three latent membrane proteins (LMP1, $-2 \mathrm{~A},-2 \mathrm{~B})$, two non-translated RNAs (EBER1 and EBER2) and several microRNAs, ${ }^{23,24}$ but only EBNA1 is regularly detected in all EBV-associated malignancies. ${ }^{24}$ Although unable to trigger cell proliferation and lacking tumorigenic potential in animal models, ${ }^{25,26}$ EBNA1 was shown to induce genomic instability in malignant B cells, as manifested by the occurrence of non-clonal chromosomal aberrations, oxidative DNA damage and activation of the DNA damage response. $^{27,28}$

In this study, we have investigated the effect of EBV latency proteins on the maintenance of telomere integrity in virusinfected cells. We report that EBNA1 promotes telomere dysfunction via induction of oxidative DNA damage.

\section{Materials and methods}

\section{Cell lines}

The cell lines used in this investigation are listed and referenced in Supplementary Information Table S1. All cells were cultured in RPMI1640 supplemented with $10 \%$ fetal calf serum, $2 \mathrm{mM}$ 
glutamine, $100 \mathrm{U} / \mathrm{ml}$ penicillin and $0.1 \mathrm{mg} / \mathrm{ml}$ streptomycin, (Sigma-Aldrich, St Louis, MO, USA). BJAB sublines expressing EBNA1, EBNA-3A and EBNA-3C were kept in medium supplemented with $2 \mathrm{mg} / \mathrm{ml}$ geneticin (Gibco, Darmstadt, Germany), and sublines carrying the tet-off transactivator were kept in medium supplemented with $0.5 \mathrm{mg} / \mathrm{ml}$ hygromycin B (Calbiochem, Darmstadt, Germany) and either $1 \mu \mathrm{g} / \mathrm{ml}$ puromycin (Calbiochem) or $2 \mathrm{mg} / \mathrm{ml}$ geneticin (Sigma-Aldrich) for BJAB-tTAE1 and BJAB-tTALMP1, respectively. Expression of the viral proteins was repressed by culture in medium containing $2 \mu \mathrm{g} / \mathrm{ml}$ doxycycline (Sigma-Aldrich). Endogenous reactive oxygen species (ROS) levels were measured by DCFDA staining, ${ }^{29}$ and quenching was achieved by culturing the cells in medium containing 3.5 $\mu \mathrm{M}$ Ebselen (Alexis Biochemicals, San Diego, CA, USA) for 1 week.

\section{Immunoblotting}

Cell lysates were prepared in lithium dodecyl sulfate sample buffer, fractionated in precast 4-12\% NUPAGE gels (Invitrogen, Carlsbad, CA, USA) and transferred to polyvinylidene fluoride membranes (Millipore, Bedford, MA, USA). The blots were probed with antibodies to EBNA1 (OT1x mouse monoclonal antibody 1:1000, kind gift of Jaap Middeldorp, Vrije Universiteit, Amsterdam, The Netherlands), EBNA-3A (rabbit serum 1:1000, ASLA, Riga, Latvia), EBNA-3C (mouse monoclonal 1:5000, kind gift of Martin Rowe, University of Birmingham, Birmingham, UK), LMP1 (S12 hybridoma supernatant 1:10000), TRF1, hTERT (sc.56807 1:1000, sc-7212 1:250, Santa Cruz Biotechnology, Santa Cruz, CA, USA), TRF2, Tankyrase, POT1, Tin2 (IMG124A 1:1000, IMG-146 1:500, IMG-5343A 1:1000, IMG-282 1:500 IMGENEX, San Diego, CA, USA), TPP1 (ab39042 1:500, Abcam, Cambridge, MA, USA) followed by the appropriate HRP-conjugates secondary antibody (Zymed, San Francisco, CA, USA), developed by enhanced chemiluminescence and analyzed using the Fuji LAS 1000 system (FujiFilm Medical Systems Inc., Stamford, CT, USA).

\section{Quantitative Fluorescence in situ hybridization (Q-FISH)}

Exponentially growing cells were treated with $30 \mathrm{ng} / \mathrm{ml}$ colcemide (KaryoMAX Invitrogen) for $90 \mathrm{~min}$ to induce metaphase arrest, washed in hypotonic buffer containing $75 \mathrm{mM} \mathrm{KCl}$ (Sigma-Aldrich), fixed in methanol/acetic acid (3:1) and dropped onto cold glass slides. Telomeres were visualized using the Telomere PNA FISH kit, (DAKO, Glostrup, Denmark). The slides were fixed in $3.7 \%$ formaldehyde (Merck, Darmstadt, Germany) for $10 \mathrm{~min}$ and washed twice in Tris-buffered saline, treated with proteinase $\mathrm{K}$ for $10 \mathrm{~min}$ at room temperature and dehydrated by consecutive $2 \mathrm{~min}$ incubations in 70,85 and $100 \%$ cold ethanol. After air-drying, hybridization solution containing the peptide nucleic acid probe was added followed by heat denaturation for $5 \mathrm{~min}$ at $80^{\circ} \mathrm{C}$ and incubation for $2 \mathrm{~h}$ at room temperature in the dark. The slides were then overlaid with washing solution for $5 \mathrm{~min}$ at $65^{\circ} \mathrm{C}$, dehydrated and mounted with 4',6-diamidino-2-phenylindole-containing Vectashield (Vector Laboratories, Inc., Burlingame, CA, USA). Digital images were captured using a LEITZ-BMRB fluorescence microscope (Leica, Wetzlar, Germany) equipped with a CCD camera (Hamamatsu Photonics, Japan) and analyzed using Adobe Photoshop (Adobe Photoshop CS4; Adobe systems Inc., San Jose, CA, USA). Chromosomes were counted and the fluorescence intensity of each telomeric signal was scored using the ImageJ software (http://rsb.info.nih.gov). The results are expressed as integrated density $\left(\mathrm{ID}=50 \mathrm{pix} / \mathrm{inch}^{2}\right)$ of telomere signals in 25 randomly selected metaphase plates from three independent experiments.

\section{Detection of TIFs and APBs by immunofluorescence-FISH (IF-FISH)}

Cells $\left(3 \times 10^{4}\right)$ in $0.1 \mathrm{ml}$ phospahte-buffered saline were deposited on glass slides by cytospin centrifugation, fixed in fresh $3.7 \%$ formaldehyde for $10 \mathrm{~min}$ and permeabilized with $0.1 \%$ Triton X-100 for $5 \mathrm{~min}$. After blocking with 3\% fetal calf serum for $30 \mathrm{~min}$, the slides were incubated for $1 \mathrm{~h}$ at room temperature with antibodies to PML (mouse monoclonal antibody1:100, kind gift of Hugues de Thé, University of Paris, Paris, France), phosphorylated histone 2AX (pH2AX) (05-636 1:100, Upstate Biotechnology, CA, USA) or 53BP1 (612523 1:100, BD Biosciences, NJ, USA) followed by Alexa Fluor 555-conjugated donkey antimouse antibodies (Invitrogen) for $30 \mathrm{~min}$. After washing and, fixation with $2 \%$ formaldehyde for $10 \mathrm{~min}$ quantitative fluorescent in situ hybridization (Q-FISH) was performed as described above.

\section{Detection of telomere sister chromatid exchanges by chromosome orientation-FISH (CO-FISH)}

The cells were grown in medium containing $10 \mu \mathrm{M} B r d U / B r d C$ (3:1) (BrdU, Sigma-Aldrich and BrdC, MP Biomedicals, Illkirch, France) for $16 \mathrm{~h}$ and chromosome slides were prepared as described. After treatment with $0.5 \mathrm{mg} / \mathrm{ml}$ RNAase A (Invitrogen) for $10 \mathrm{~min}$ at $37^{\circ} \mathrm{C}$, staining with $0.5 \mu \mathrm{g} / \mathrm{ml}$ Hoechst 33258 in $2 \times$ SSC for $15 \mathrm{~min}$ at room temperature and crosslinking by exposure to 365-nm UV light (Stratalinker 1800, Agilent Technologies, St Clara, CA, USA) for $30 \mathrm{~min}$, the slides were digested with $10 \mathrm{U} / \mu \mathrm{l}$ Exonuclease III (Fermentas, Leon-Rot, Germany) for $10 \mathrm{~min}$ at room temperature and sequentially incubated with TAMRA-TelG 5'-(TTAGGG)3-3' and FITC-TelC $5^{\prime}$-[CCCTAA]3-3' probes (Cambridge Research Biomedicals, Cleveland, UK) at room temperature for $2 \mathrm{~h}$.

\section{Quantification of telomere-associated TRF2}

Cytospin slides were permeabilized with $0.1 \%$ Triton X-100 followed by fixation in fresh $2 \%$ paraformaldehyde (SigmaAldrich) for $20 \mathrm{~min}$, blocking with $2 \%$ bovine serum albumin (BSA) for $30 \mathrm{~min}$ and overnight incubation at $4{ }^{\circ} \mathrm{C}$ with the antiTRF2 antibody. Q-FISH was then performed as described. Digital images were analyzed with the Volocity Software (Improvision, University of Warwick Science Park, England). Telomeres (red fluorescence) were identified in hundred nuclei and the average intensity of red (telomere) and green (TRF2) fluorescence within each selected area was quantified.

\section{Statistical analysis}

Statistical analysis was performed using Student's $t$-test. $P$-values $\leqslant 0.05$ were considered as significant.

\section{Results}

Telomere aberrations in EBNA1-expressing cells Previous studies demonstrating the presence of abnormal telomeres and chromosomal aberrations in a restricted panel of EBV-positive and -negative Burkitt's Lymphoma lines suggested that EBV carriage may be associated with telomere dysfunction. ${ }^{27}$ To verify this observation, the analysis was extended to a larger panel of paired cell lines including in vitro 
A
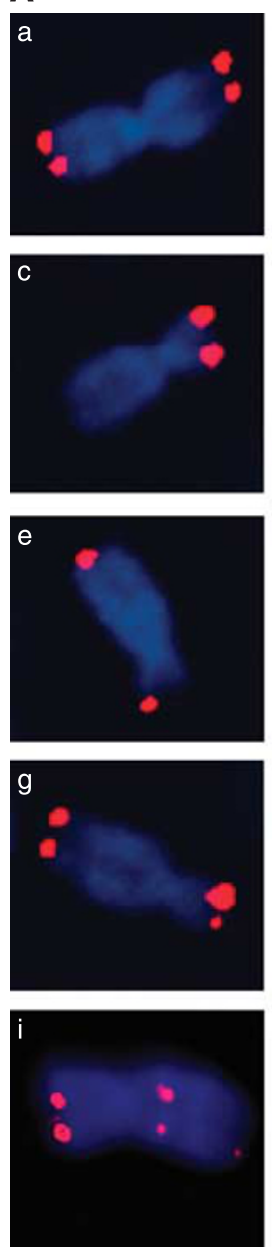
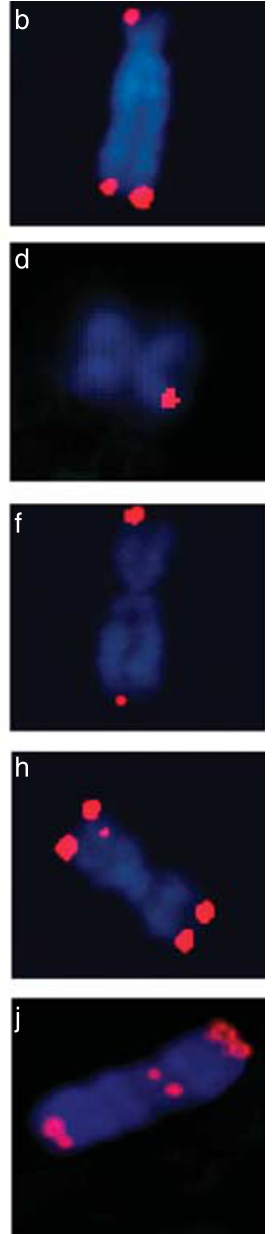

B

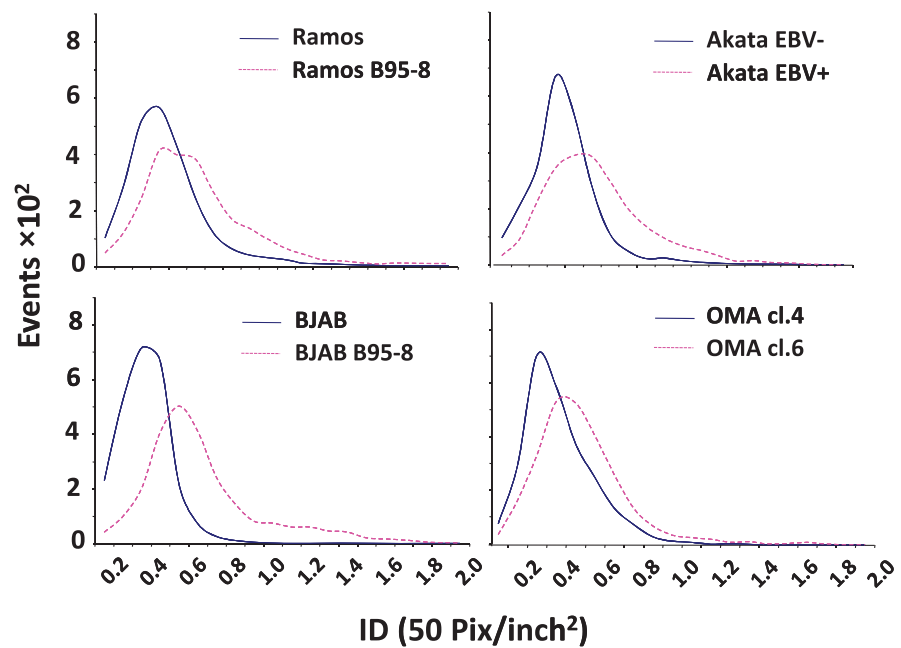

C

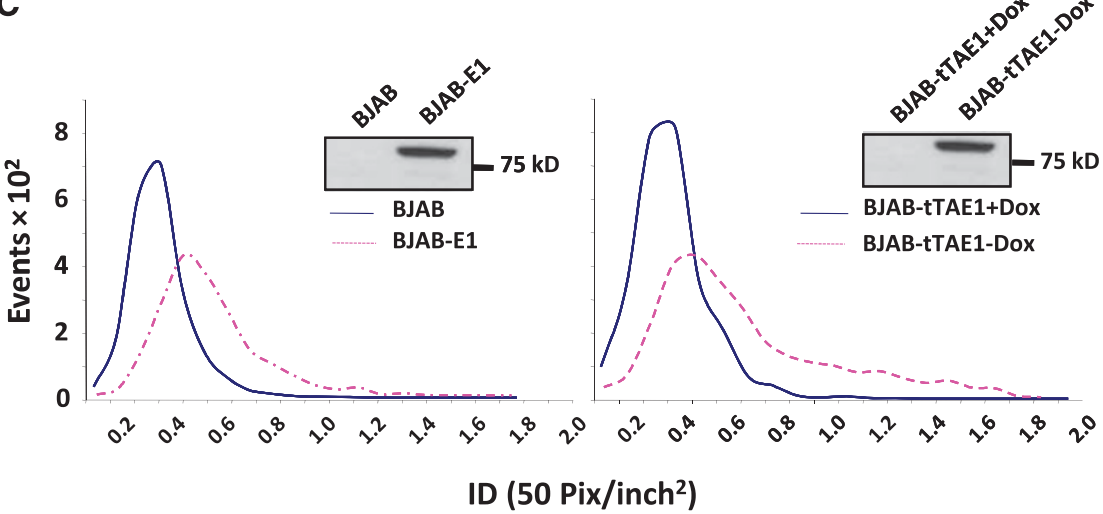

Figure 1 EBNA1 expression induces telomere aberrations and telomere length heterogeneity. (A) Representative micrographs of BJAB-E1 metaphase chromosomes illustrating the telomere aberrations scored in EBV-positive and -negative cell lines: (a) chromosome with four telomere signals of equal intensity; (b-d) chromosomes lacking one or more telomere signal; (e, f) chromosomes with fused telomeres; (g) chromosome with telomere signals of different intensity; $(\mathbf{h}-\mathbf{j})$ chromosomes with interstitial telomeres. (B) Quantification of telomere signal intensity in pairs of EBV-negative and -positive cell lines. The telomeres were detected by quantitative (Q)-FISH in three independent experiments, each including 25 randomly selected metaphases and signal intensity represented as integrated pixel density (ID, 50 pix/inch ${ }^{2}$ ) was quantified using the Image software. (C) Intensity of telomere signals detected by Q-FISH in BJAB sublines expressing stable (BJAB-E1, left panel) or inducible EBNA1 (BJAB-tTAE1, right panel). BJAB-tTAE1 cells were kept in the presence or absence of doxycycline for 1 month before the assay. EBNA1 expression was confirmed in western blots probed with the OT1x antibody (inserts).

EBV-converted sublines of the EBV-negative Ramos and BJAB and EBV genome-loss variants of the EBV-positive Burkitt's Lymphoma lines Akata and Oma. Representative micrographs illustrating the type of telomere aberrations recorded in Q-FISHstained metaphase plates are shown in Figure $1 \mathrm{~A}$ and a compilation of the frequency of these aberrations in paired EBV-positive and-negative cell lines is presented in Supplementary Information Figure S1A. Although four clearly distinguishable telomere signals of similar intensities were detected in the majority of chromosomes from the EBV-negative BJAB, Ramos, Akata (-) and Oma cl.4 (Figure 1A, panel a, Supplementary Figure S1A), more than $40 \%$ of the chromosomes in the EBVcarrying cell lines showed less than four telomere signals (Figure 1A panels b, c, d), fused telomeres (Figure 1A panels e, $\mathrm{f})$, telomeres of widely different fluorescence intensity (Figure $1 \mathrm{~A}$, panels $\mathrm{b}, \mathrm{f}, \mathrm{g}$ ) or interstitial telomeres (Figure 1A, panels $h, i, j)$. Quantification of the intensity of individual telomere signals in three independent experiments, each including 25 randomly selected metaphases, revealed a cellline specific variation in total signal that was only marginally influenced by the presence of EBV (Figure 1B). However, although a narrow distribution of signal intensity was regularly observed in the EBV-negative cell lines, EBV carriage was associated with a remarkable broadening of the signal intensity distribution, confirming the increased heterogeneity of telomere size detected by visual inspection of the micrographs.

In order to investigate which EBV latent protein might be responsible for this phenotype, the distribution of telomere signal intensity was compared in the EBV-negative $B J A B$ and in transfected sublines stably expressing EBNA1, $-3 \mathrm{~A},-3 \mathrm{C}$ and LMP1. Expression of the viral proteins was in each case confirmed in western blots probed with specific antibodies (Figure 1C and Supplementary Information Figure S1B inserts). A significant broadening of telomere signal distribution was observed in the EBNA1 transfectant (Figure 1C, left panel), whereas expression of EBNA-3A, -3C and LMP1 had no effect (Supplementary Information Figure S1B). The effect of EBNA1 was confirmed by visual scoring of chromosome-associated telomere signals, which revealed a significant increase in the number of chromosomes with abnormal telomeres 


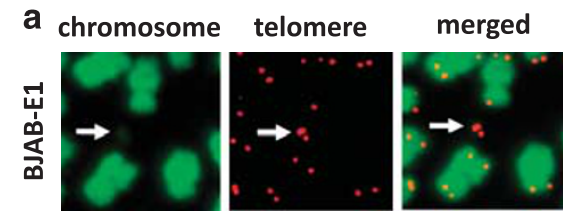

b
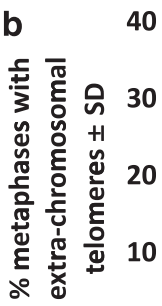

40
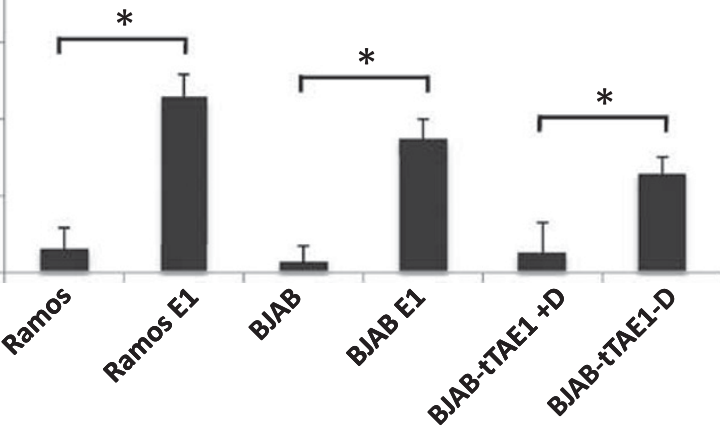

d

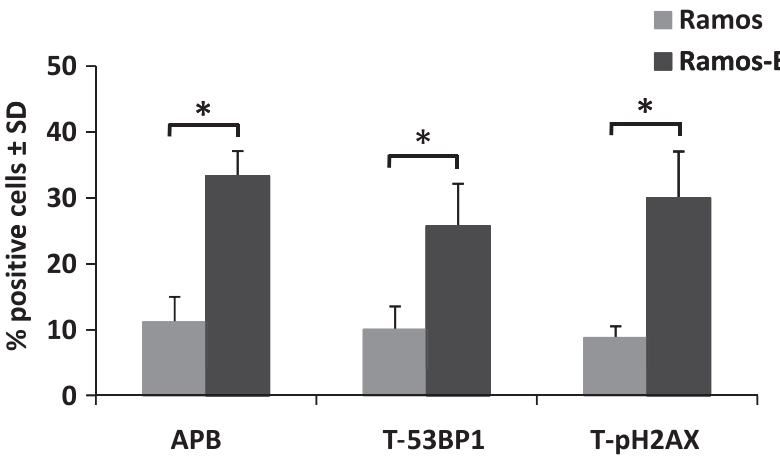

C
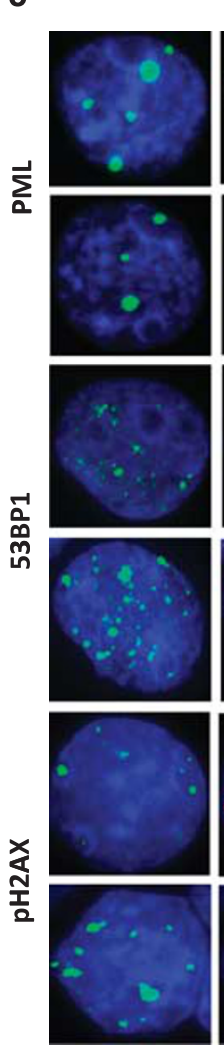

merged

enlarged

E1
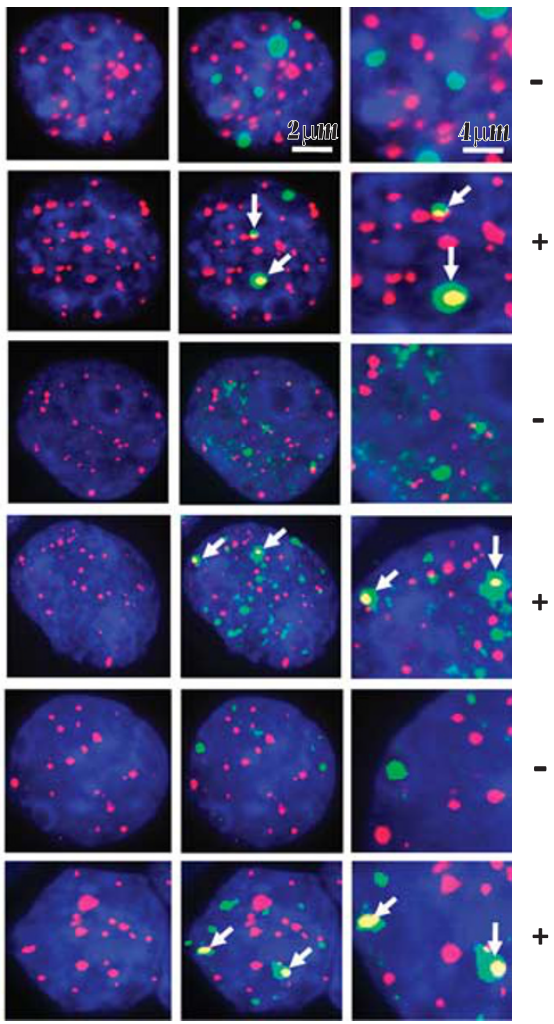

Figure 2 EBNA1 expression induces telomere dysfunction. (a) Representative Q-FISH illustrating the occurrence of extra-chromosomal telomeric signals (arrows) in BJAB-E1. The chromosomes were stained with 4',6-diamidino-2-phenylindole (DAPI). (b) Mean \pm s.d. of extrachromosomal telomere positive cells scored in 25 metaphase spreads of EBNA1-positive and-negative cell lines in three independent experiments. ${ }^{*} P \leqslant 0.01$. (c) Representative immunofluorescence-FISH (IF-FISH) micrographs illustrating the presence of APBs (upper panels), 53BP1 (middle panels) and $\mathrm{pH} 2 \mathrm{AX}$ (lower panels) foci in Ramos and Ramos-E1. PML, 53BP1 and pH2AX immunofluorescence in green and telomeres Q-FISH in red. The nuclei were stained by DAPI (blue). (d) Compilation of the \% of cell containing one or more telomeric APB, pH2AX and 53BP1 foci in EBNA1positive and-negative Ramos in three independent experiments in which at least 25 nuclei were scored. ${ }^{*} P \leqslant 0.01$.

(Supplementary Information Figure S1C). A comparable broadening of telomere signal intensity was observed within 4 weeks of doxycycline withdrawal in the BJAB-tTAE1 cell line that carries a conditional EBNA1 (Figure 1C, right panel). Thus, EBNA1 expression is associated with several morphological signs of telomere dysfunction including loss or gain of telomere signals and wide heterogeneity of telomere length.

\section{EBNA1 induces telomere dysfunction}

Telomere dysfunction is caused by the impairment of control mechanisms that maintain telomere integrity, which leads to telomere breaks and activation of telomere-specific DNA damage responses attempting to repair the damage. ${ }^{30}$ Imprecise repair results in the accumulation of extrachromosomal telomeric DNA repeats, principally in the form of t-circles. ${ }^{18}$ As illustrated by representative Q-FISH of metaphase plates from BJAB-E1 cells (Figure 2a) and summarized in Figure 2b, a significant increase of extrachromosomal telomeric DNA was observed in stably transfected BJAB and Ramos and was induced upon doxycycline withdrawal in the BJAB-tTAE1 cell line, confirming that expression of EBNA1 is associated with DNA breakage at telomeres.
DNA damage response factors such as $\mathrm{pH} 2 \mathrm{AX}$ and 53BP1 are recruited at dysfunctional telomeres in nuclear foci known as telomere dysfunction-induced foci (TIFs), ${ }^{12}$ and the activation of DNA repair is associated with the appearance of telomereassociated promyelocytic leukemia nuclear bodies (APBs) in which PML colocalizes with telomeric DNA and telomereassociated proteins. ${ }^{16}$ IF-FISH staining for telomeres and $\mathrm{pH} 2 \mathrm{AX}, 53 \mathrm{BP} 1$ or PML was performed in EBNA1-positive and -negative Ramos cells. Examination of one focal plane per nucleus revealed a significant increase of $\mathrm{pH} 2 \mathrm{AX}, 53 \mathrm{BP} 1$ and PML foci in the EBNA1-positive cells (Figure 2c, left panel) and dual staining confirmed that many of these foci colocalize with telomeres (Figure 2c, merged). A compilation of the results obtained in three independent experiments is shown in Figure 2d. A comparable EBNA1-associated increase of TIFs and APBs was observed in the BJAB-E1 and BJAB-tTAE1 cell lines (data not shown), although the possibility of chance colocalization could not be unequivocally excluded in these cells because of the small size of telomeres detected by Q-FISH in interphase nuclei.

Telomere-sister chromatid exchange is often observed in cells with damaged or eroded telomeres. ${ }^{31}$ Analysis of telomere-sister chromatid exchange by chromosome orientation (CO)-FISH 
a
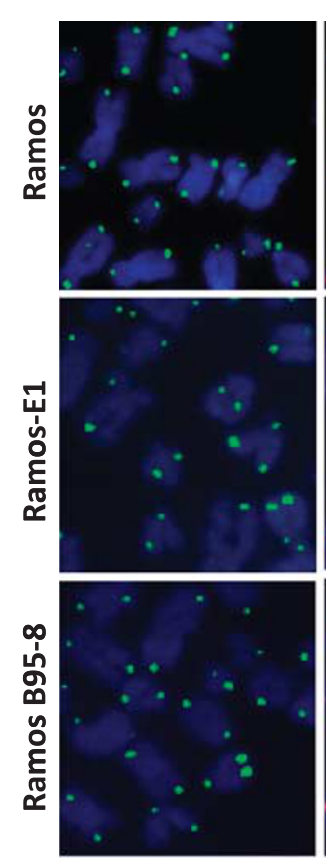

TelC
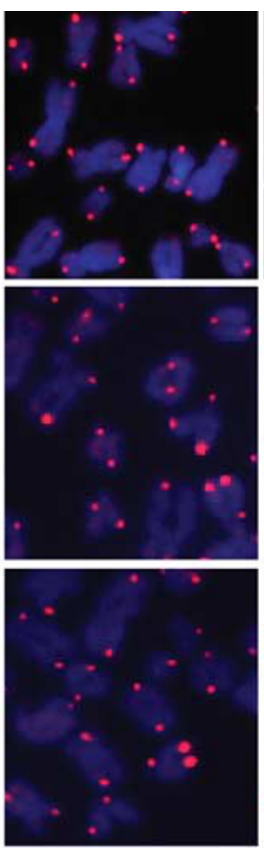

Merged
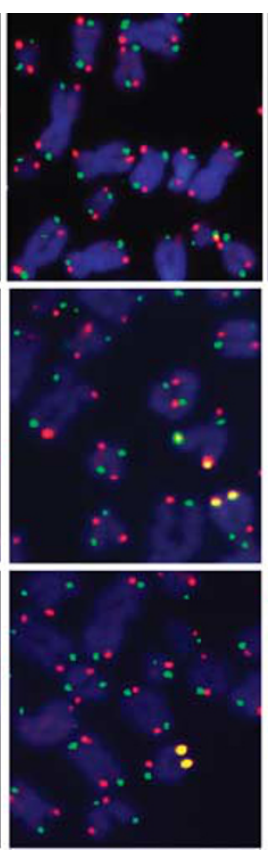

enlarged
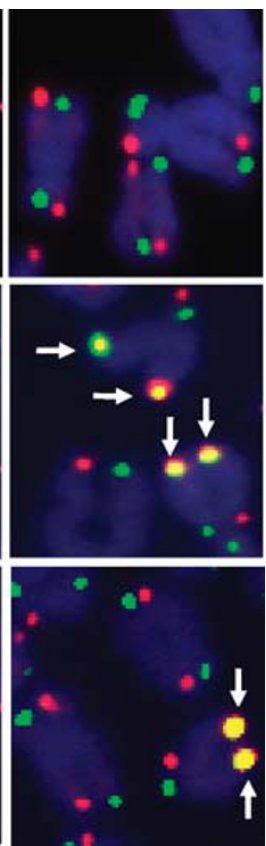

b

\begin{tabular}{|c|c|c|c|c|}
\hline & Ch-Plate & Ch-Number & T-SCE & $\%$ \\
\hline Ramos & 19 & 910 & 18 & 1,98 \\
\hline Ramos-E1 & 19 & 813 & 86 & 10,58 \\
\hline Ramos-B95-8 & 19 & 884 & 77 & 9,28 \\
\hline
\end{tabular}

Figure 3 Expression of EBNA1 is associated with enhanced telomere-sister chromatid exchange. (a) Representative micrographs illustrating the occurrence of telomere-sister chromatid exchange in EBV-converted and EBNA1-transfected Ramos. Chromosome orientation-FISH (CO-FISH) was performed with the telomere strand-specific probes TelC (leading strand template, red) and TelG (lagging strand template, green), telomeresister chromatid exchange is visualized by colocalization of red and green signals on the same telomere (arrows). (b) Compilation of the results obtained in two independent CO-FISH experiments. A fivefold increase of telomere-sister chromatid exchange-positive chromosomes was detected in EBV-converted and EBNA1-transfected Ramos cells.

using G-rich (lagging strand template, Figure 3a, left panels) and C-rich (leading strand template, Figure 3a middle panel) probes demonstrated a significant fivefold increase of telomere-sister chromatid exchange in EBV-positive Ramos compared with the EBV-negative parental and a similar increase was observed in the Ramos-E1 transfectant, confirming that EBNA1 expression is sufficient for the effect (Figures $3 a$ and b). Collectively these findings demonstrate that EBNA1 expression is associated with the induction of telomere dysfunction as detected by extrachromosomal telomere signals and accumulation of TIFs, APBs and telomeric-sister chromatid exchanges.

\section{EBNA1 promotes telomere uncapping via induction of oxidative stress}

Telomere uncapping is a common cause of DNA damage at telomeres. ${ }^{32}$ In order to assess whether telomere deprotection might explain the telomere dysfunction phenotype induced by EBNA1, we first investigated the expression of components of the shelterin complex and other telomere-associated proteins. To this end, western blots of cell lysates from paired EBNA1negative and -positive cell lines were sequentially probed with appropriately selected cocktails of antibodies specific for the DNA-binding subunits, TRF1, TRF2 and POT1, associated components of the shelterin complex, TPP1 and TIN2, and the telomere homeostasis-related proteins TANK and TERT. Representative western blots are shown in Figure 4a. The expression of EBNA1 was not associated with regular changes in the intensity of the specific bands, although minor differences in the expression levels of individual protein were occasionally observed. Similar results were obtained in all the cell lines examined in this study, including EBV-converted sublines of $B J A B$ and Ramos and EBV-positive and loss variants of Akata and Oma Burkitt's Lymphomas (data not shown).

Binding of TRF2 is essential for telomere protection. ${ }^{7}$ Having ascertained that EBNA1 does not influence the expression of TRF2, we then asked whether the localization of TRF2 might be affected. Telomeres and TRF2 were visualized in EBNA1positive and -negative cells by IF-FISH and the amount of telomere-associated TRF2 was quantified. Representative images illustrating the colocalization of TRF2 (green fluorescence) and telomere signals (red fluorescence) in Ramos and Ramos-E1 are shown in Figure 4b. A striking decrease in the intensity of TRF2 signals associated with telomere was observed in EBNA1-positive cells in which several telomeres appeared to lack any associated TRF2 fluorescence (indicated by arrows). Quantification of telomere fluorescence intensity in 100 nuclei confirmed that EBNA1 expression was associated with a broadening of the telomere signal distribution (Figure 4c, horizontal axis) and with a significant decrease in the amount of telomere-associated TRF2 fluorescence (Figure 3c, vertical axis and Figure $4 d$ ). Similar results were obtained on comparison of the BJAB and BJAB-E1 cell lines (data not shown). 

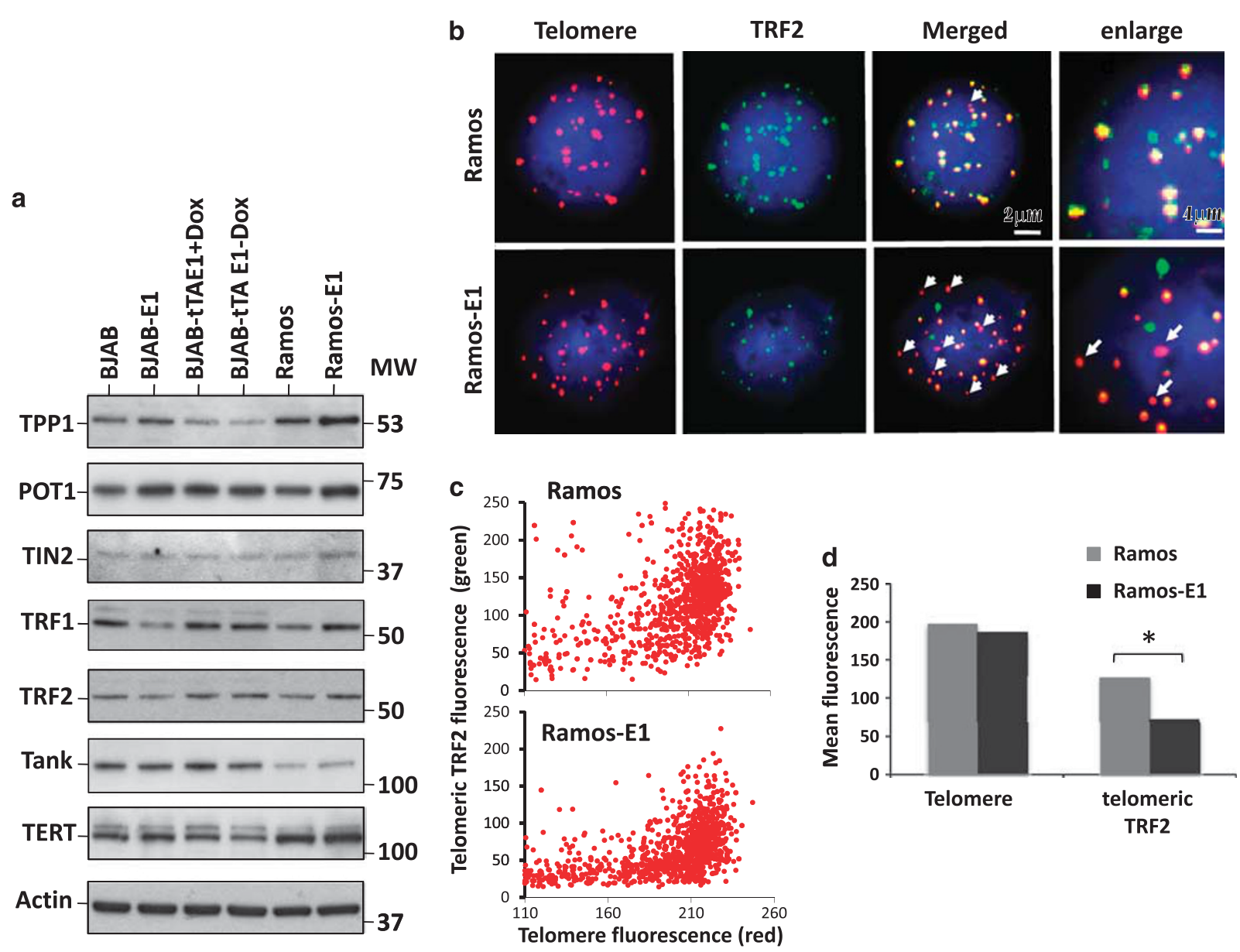

Figure 4 EBNA1 induces telomere deprotection by displacement of TRF2. (a) Representative western blot illustrating the expression of shelterin and telomere proteins in EBNA1-positive and -negative BJAB and Ramos. Total cell lysates were fractioned by SDS-PAGE, and blots were sequentially probed with cocktails of the indicated antibodies. (b) Decrease of telomere-associated TRF2 in EBNA1-expressing cells. Representative micrographs illustrating the colocalization of TRF2 (green) and telomere (red) signals in Ramos and Ramos-E1. Telomeres lacking colocalized green fluorescence are indicated by arrows. (c) Quantification of telomere and telomere localized TRF2 signals. The cells were stained by immunofluorescence-FISH (IF-FISH) and telomeres and TRF2 were identified by means of red and green fluorescence in 100 nuclei. (d) The intensity of green fluorescence overlapping with each red telomere was quantified using the Volocity software. One representative experiment out of three is shown in the figure.

We have previously shown that EBNA1 induces DNA damage in B lymphocytes through transcriptional upregulation of the NADPH oxidase NOX2 and consequent accumulation of ROS. ${ }^{28}$ We therefore asked whether the telomere dysfunction phenotype could be attributed to the induction of ROS. We first tested whether oxidative stress induces TIFs and APBs in EBVnegative cells. In accordance with previous reports demonstrating that mild oxidative stress induces telomere erosion, ${ }^{33}$ short treatment of EBV-negative Ramos with 10 or $50 \mu \mathrm{M} \mathrm{H}_{2} \mathrm{O}_{2}$ induced levels of TIFs and APBs comparable to those observed in the Ramos-E1 transfectant (Supplementary Information Figure $\mathrm{S} 2 \mathrm{~A}$ ). We then sought to establish a link between telomere dysfunction and the increased endogenous levels of ROS in EBNA1-expressing cells. To this end, Ramos-E1 cells were cultured for 1 week in a medium containing $3.5 \mu \mathrm{M}$ of the ROS scavenger glutathione peroxidase mimetic Ebselen before detection of APBs by IF-FISH. As previously reported, ${ }^{28}$ Ebselen treatment was associated with a significant decrease in the levels of ROS measured by DCFDA staining (data not shown) and with virtually complete disappearance of $\mathrm{pH} 2 \mathrm{AX}$ foci (Figure 5a, upper panel). This correlated with a significant reduction of APBs (Figure 5 a lower panel and Figure 5b), indicating that ROS-dependent DNA damage is critically involved in the induction of telomere dysfunction in EBNA1expressing cells. We then asked whether the capacity of Ebselen to inhibit the formation of APBs was also associated with restoration of telomere protection as measured by telomeric localization of TRF2. To this end, telomeres and TRF2 were detected by IF-FISH in Ramos and Ramos-E1, and the intensity of telomere colocalized TRF2 signals was quantified. As illustrated by the representative experiments shown in Figure 5c and summarized in Supplementary Information Figure $\mathrm{S} 2 \mathrm{~B}$, treatment with Ebselen resulted in reconstitution of telomere-associated TRF2 fluorescence to levels comparable to those observed in the EBV-negative Ramos cell line. Collectively, these results support the conclusion that oxidative stress mediates the induction of telomere dysfunction through displacement of the shelterin protein TRF2.

\section{Discussion}

EBV is a human tumor virus and a potent growth-transforming agent for human B cells. Virus carriage is associated with increased genomic instability in B-cell lymphomas. ${ }^{27}$ In this study, we have documented the occurrence of telomere 


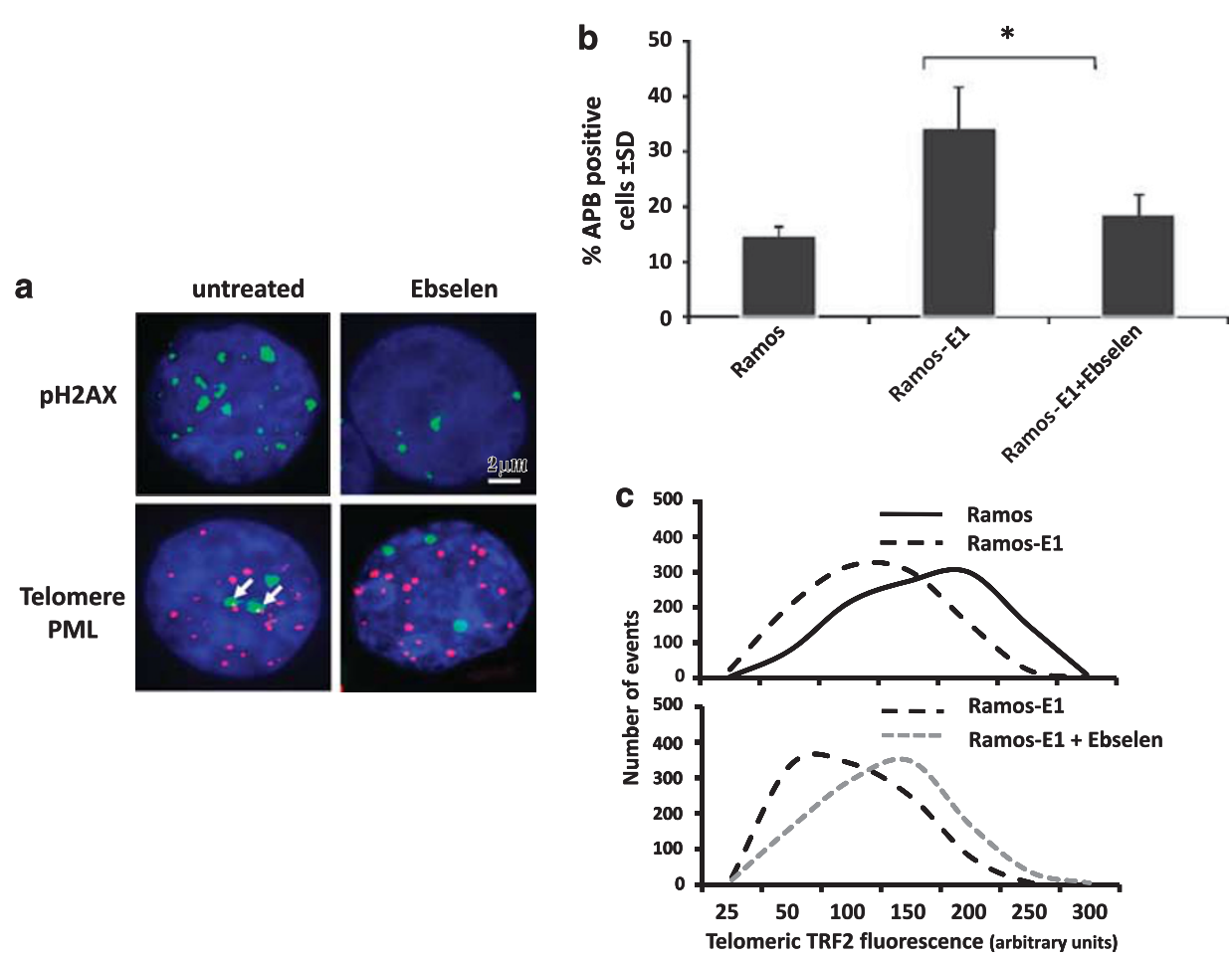

Figure 5 EBNA1 promotes telomere dysfunction via induction of ROS. Ramos and Ramos-E1 were cultured in the presence of the ROS scavenger Ebselen for 1 week before the assays. (a) Representative micrographs illustrating the effect of Ebselen on the presence of pH2AX foci (upper panel) and APBs detected by PML (green) and telomere (red) colocalization (lower panel, arrows). (b) Quantification of APBs in control and Ebselen treated Ramos-E1. Mean \pm s.d. of three experiments. ${ }^{*} P \leqslant 0.01$ (c) Ebselen treatment promotes the relocalization of TRF2 at telomere. Quantification of telomere and telomere localized TRF2 signals was performed as described in the legend of Figure 4c. One representative experiment out of three is shown in the figure.

dysfunction in EBV-infected cells and identified EBNA1 as the viral protein causing this phenotype via induction of oxidative stress-dependent telomere uncapping.

Numerous signs of telomere dysfunction accumulate in B-cell lines upon stable or inducible expression of EBNA1, including a striking heterogeneity in telomere length (Figure 1), a significant increase of extrachromosomal telomeric DNA, accumulation of DNA damage response and repair proteins at telomeres (Figure 2), and an approximately fivefold increase of telomeresister chromatid exchange (Figure 3). Failure of the machinery that controls telomere capping is a major trigger of telomere dysfunction in proliferating cells. ${ }^{30}$ Thus, loss of TRF2 in cells expressing dominant-negative mutants or specific small hairpin RNAs, and deletion of POT1 were shown to induce activation of the DNA damage response, aberrant chromosome recombination and genomic instability. 7,34 The effect of EBNA1 on the accumulation of TIFs containing DNA-damage response proteins (Figure 2) was paralleled by the displacement of TRF2 from telomeres (Figure 4). It is noteworthy that the levels of TRF2 detected by western blot in total cell lysates were not significantly changed, which may reflect the selective increase of an extra-telomeric TRF2 pool. ${ }^{35}$ TRF2 was shown to cooperatively bind with EBNA1 to the origin of viral replication OriP $P^{36}$ and may be recruited to other EBNA1-binding sites on cellular DNA. However, we have failed to demonstrate any interaction of TRF2 with EBNA1 by co-immunoprecipitation and could not convincingly demonstrate localization of EBNA1 at telomeres.

The uncertain fate of the displaced TRF2 notwithstanding, the accumulation of telomeric foci of DNA-damage response proteins strongly supports the involvement of telomere deprotection in the telomere dysfunction phenotype induced by
EBNA1. Our previous finding that EBNA1 induces oxidative DNA damage and genomic instability by transcriptional upregulation of the catalytic subunit of the NADPH oxidase NOX2 ${ }^{28}$ provided an important insight on the possible mechanism of this effect. Oxidative stress causes telomere dysfunction, ${ }^{37}$ which is at least partly due to a diminished binding of TRF1 and TRF2 to oxidized telomeric DNA. ${ }^{33}$ We have found that, although mild oxidative stress induced levels of TIFs and APBs comparable to those induced by EBNA1 expression in EBV-negative cells (Supplementary Information Figure S2), the formation of TIFs and APBs was inhibited in EBNA1-positive cells when the endogenous levels of ROS were decreased by treatment with Ebselen (Figure 5). Most importantly, this was associated with relocalization of TRF2 at telomeres, establishing a direct correlation between the oxidative stress induced by EBNA1 and telomere uncapping.

The telomere abnormalities associated with EBNA1 expression are characteristic features of ALT, a mechanism that maintains telomere homeostasis in a subset of malignant cells with poor or absent telomerase activity. ${ }^{17}$ Previous reports demonstrating that EBV conversion does not alter the activity of telomerase in B-lymphoma lines ${ }^{38}$ speak against a direct effect of EBNA1 on telomerase, which is also supported by our failure to observe changes in the expression of TERT and other telomere-associated proteins in cells expressing stable or inducible EBNA1 (Figure 4). It is noteworthy that persistent telomerase activity may not preclude the activation of recombination-dependent mechanisms for telomere elongation as ALT was shown to remain active upon reconstitution of telomerase activity in ALT-positive cell lines. ${ }^{39,40}$ The possibility that EBV may activate telomerase-independent mechanisms for maintenance of telomere homeostasis is line with the observations 
that the average length of telomeres remains relatively constant during the early phases of EBV-induced growth transformation, ${ }^{41,42}$ whereas a robust upregulation of telomerase activity is only detected after several weeks or months in culture. ${ }^{41}$ Furthermore, some EBV immortalized lymphoblastoid cell lines overcome a growth crisis after approximately 150 passages in vitro in the absence of detectable telomerase activity. ${ }^{43}$ Conceivably, if EBV activates ALT, this could rescue the infected cells from replicative senescence during the early phase of B-cell immortalization. The inherently imprecise recombination mechanisms that underlie ALT may also give rise to telomeres too short for efficient capping and therefore exposed to inappropriate repair and chromosomal aberration. This scenario is in line with the high frequency of non-clonal chromosomal aberrations in EBNA1-expressing B-lymphoma lines, ${ }^{27}$ and is also supported by the recent findings of Lacoste et al. ${ }^{42}$ who, although monitoring the evolution of lymphoblastoid cell lines from the time of EBV infection, observed high karyotypic instability during the first 10-25 weeks in culture followed by stabilization approximately at the time when a significant upregulation of telomerase activity would be expected.

\section{Conflict of interest}

The authors declare no conflict of interest.

\section{Acknowledgements}

We are deeply grateful to Hughes de Thé, Jaap Middeldorp and Martin Rowe for the kind gift of antibodies and cell lines. This study was supported by grants awarded by the Swedish Cancer Society, the Swedish Medical Research Council and Karolinska Institutet, Stockholm, Sweden and by the European Community Integrated Project on Infection and Cancer, INCA, Project no. LSHC-CT-2005-018704. SAK was supported by a fellowship awarded by the Iranian Government.

\section{References}

1 Rudolph KL, Millard M, Bosenberg MW, DePinho RA. Telomere dysfunction and evolution of intestinal carcinoma in mice and humans. Nat Genet 2001; 28: 155-159.

2 Artandi SE, Chang S, Lee SL, Alson S, Gottlieb GJ, Chin L et al. Telomere dysfunction promotes non-reciprocal translocations and epithelial cancers in mice. Nature 2000; 406: 641-645.

3 Moyzis RK, Buckingham JM, Cram LS, Dani M, Deaven LL, Jones $\mathrm{MD}$ et al. A highly conserved repetitive DNA sequence, (TTAGGG)n, present at the telomeres of human chromosomes. Proc Natl Acad Sci USA 1988; 85: 6622-6626.

4 de Lange T. Shelterin: the protein complex that shapes and safeguards human telomeres. Genes Dev 2005; 19: 2100-2110.

5 Makarov VL, Hirose Y, Langmore JP. Long G tails at both ends of human chromosomes suggest a $\mathrm{C}$ strand degradation mechanism for telomere shortening. Cell 1997; 88: 657-666.

6 Griffith JD, Comeau L, Rosenfield S, Stansel RM, Bianchi A, Moss $\mathrm{H}$ et al. Mammalian telomeres end in a large duplex loop. Cell 1999; 97: 503-514.

7 van Steensel B, Smogorzewska A, de Lange T. TRF2 protects human telomeres from end-to-end fusions. Cell 1998; 92: 401-413.

8 van Steensel B, de Lange T. Control of telomere length by the human telomeric protein TRF1. Nature 1997; 385: 740-743.

9 Smith S, de Lange T. Cell cycle dependent localization of the telomeric PARP, tankyrase, to nuclear pore complexes and centrosomes. J Cell Sci 1999; 112 (Part 21): 3649-3656.

10 Shay JW, Pereira-Smith OM, Wright WE. A role for both RB and p53 in the regulation of human cellular senescence. Exp Cell Res 1991; 196: 33-39.
11 Karlseder J, Smogorzewska A, de Lange T. Senescence induced by altered telomere state, not telomere loss. Science 2002; 295: 2446-2449.

12 Takai H, Smogorzewska A, de Lange T. DNA damage foci at dysfunctional telomeres. Curr Biol 2003; 13: 1549-1556.

13 Shay JW, Bacchetti S. A survey of telomerase activity in human cancer. Eur J Cancer 1997; 33: 787-791.

14 Bryan TM, Englezou A, Gupta J, Bacchetti S, Reddel RR. Telomere elongation in immortal human cells without detectable telomerase activity. EMBO J 1995; 14: 4240-4248.

15 Bryan TM, Englezou A, Dalla-Pozza L, Dunham MA, Reddel RR. Evidence for an alternative mechanism for maintaining telomere length in human tumors and tumor-derived cell lines. Nat Med 1997; 3: 1271-1274.

16 Yeager TR, Neumann AA, Englezou A, Huschtscha LI, Noble JR, Reddel RR. Telomerase-negative immortalized human cells contain a novel type of promyelocytic leukemia (PML) body. Cancer Res 1999; 59: 4175-4179.

17 Dunham MA, Neumann AA, Fasching CL, Reddel RR. Telomere maintenance by recombination in human cells. Nat Genet 2000; 26: $447-450$.

18 Henson JD, Reddel RR. Assaying and investigating alternative lengthening of telomeres activity in human cells and cancers. FEBS Lett 2010; 584: 3800-3811.

19 Jiang WQ, Zhong ZH, Henson JD, Neumann AA, Chang AC, Reddel RR. Suppression of alternative lengthening of telomeres by Sp100-mediated sequestration of the MRE11/RAD50/NBS1 complex. Mol Cell Biol 2005; 25: 2708-2721.

20 Tanaka H, Mendonca MS, Bradshaw PS, Hoelz DJ, Malkas LH, Meyn MS et al. DNA damage-induced phosphorylation of the human telomere-associated protein TRF2. Proc Natl Acad Sci USA 2005; 102: 15539-15544

21 Fasching CL, Neumann AA, Muntoni A, Yeager TR, Reddel RR. DNA damage induces alternative lengthening of telomeres (ALT) associated promyelocytic leukemia bodies that preferentially associate with linear telomeric DNA. Cancer Res 2007; 67: 7072-7077.

22 Young LS, Rickinson AB. Epstein-Barr virus: 40 years on. Nat Rev Cancer 2004; 4: 757-768.

23 Xia T, O'Hara A, Araujo I, Barreto J, Carvalho E, Sapucaia JB et al. EBV microRNAs in primary lymphomas and targeting of CXCL-11 by ebv-mir-BHRF1-3. Cancer Res 2008; 68: 1436-1442.

24 Young LS, Murray PG. Epstein-Barr virus and oncogenesis: from latent genes to tumours. Oncogene 2003; 22: 5108-5121.

25 Altmann M, Pich D, Ruiss R, Wang J, Sugden B, Hammerschmidt W. Transcriptional activation by EBV nuclear antigen 1 is essential for the expression of EBV's transforming genes. Proc Natl Acad Sci USA 2006; 103: 14188-14193.

26 Kang MS, Soni V, Bronson R, Kieff E. Epstein-Barr virus nuclear antigen 1 does not cause lymphoma in C57BL/6J mice. J Virol 2008; 82: 4180-4183.

27 Kamranvar SA, Gruhne B, Szeles A, Masucci MG. Epstein-Barr virus promotes genomic instability in Burkitt's lymphoma. Oncogene 2007; 26: 5115-5123.

28 Gruhne B, Sompallae R, Marescotti D, Kamranvar SA, Gastaldello $S$, Masucci MG. The Epstein-Barr virus nuclear antigen-1 promotes genomic instability via induction of reactive oxygen species. Proc Natl Acad Sci USA 2009; 106: 2313-2318.

29 Eruslanov E, Kusmartsev S. Identification of ROS using oxidized DCFDA and flow-cytometry. Methods Mol Biol 2010; 594: 57-72.

30 Cesare AJ, Reddel RR. Telomere uncapping and alternative lengthening of telomeres. Mech Ageing Dev 2008; 129: 99-108.

31 Londono-Vallejo JA, Der-Sarkissian H, Cazes L, Bacchetti S, Reddel RR. Alternative lengthening of telomeres is characterized by high rates of telomeric exchange. Cancer Res 2004; 64: 2324-2327.

32 de Lange T. Protection of mammalian telomeres. Oncogene 2002; 21: $532-540$

33 Opresko PL, Fan J, Danzy S, Wilson III DM, Bohr VA. Oxidative damage in telomeric DNA disrupts recognition by TRF1 and TRF2. Nucleic Acids Res 2005; 33: 1230-1239.

$34 \mathrm{Wu}$ L, Multani AS, He H, Cosme-Blanco W, Deng Y, Deng JM et al. Pot1 deficiency initiates DNA damage checkpoint activation and aberrant homologous recombination at telomeres. Cell 2006; 126: $49-62$. 
35 Takai KK, Hooper S, Blackwood S, Gandhi R, de Lange T. In vivo stoichiometry of shelterin components. J Biol Chem 2010; 285: 1457-1467.

36 Deng Z, Lezina L, Chen CJ, Shtivelband S, So W, Lieberman PM. Telomeric proteins regulate episomal maintenance of Epstein-Barr virus origin of plasmid replication. Mol Cell 2002; 9: 493-503.

37 Aragona M, Maisano R, Panetta S, Giudice A, Morelli M, La Torre I et al. Telomere length maintenance in aging and carcinogenesis. Int J Oncol 2000; 17: 981-989.

38 Mochida A, Gotoh E, Senpuku H, Harada S, Kitamura R, Takahashi $\mathrm{T}$ et al. Telomere size and telomerase activity in Epstein-Barr virus (EBV)-positive and EBV-negative Burkitt's lymphoma cell lines. Arch Virol 2005; 150: 2139-2150.

39 Cerone MA, Londono-Vallejo JA, Bacchetti S. Telomere maintenance by telomerase and by recombination can coexist in human cells. Hum Mol Genet 2001; 10: 1945-1952.

40 Perrem K, Colgin LM, Neumann AA, Yeager TR, Reddel RR. Coexistence of alternative lengthening of telomeres and telomer- ase in hTERT-transfected GM847 cells. Mol Cell Biol 2001; 21: 3862-3875.

41 Counter CM, Botelho FM, Wang P, Harley CB, Bacchetti S. Stabilization of short telomeres and telomerase activity accompany immortalization of Epstein-Barr virus-transformed human B lymphocytes. J Virol 1994; 68: 3410-3414.

42 Lacoste S, Wiechec E, Dos Santos Silva AG, Guffei A, Williams G, Lowbeer $M$ et al. Chromosomal rearrangements after ex vivo Epstein-Barr virus (EBV) infection of human B cells. Oncogene 2010; 29: 503-515.

43 Sugimoto $M$, Ide $T$, Goto $M$, Furuichi $Y$. Reconsideration of senescence, immortalization and telomere maintenance of Epstein-Barr virus-transformed human B-lymphoblastoid cell lines. Mech Ageing Dev 1999; 107: 51-60.

\section{(a) This work is licensed under the Creative Commons Attribution-NonCommercial-No Derivative Works 3.0 Unported License. To view a copy of this license, visit http://} creativecommons.org/licenses/by-nc-nd/3.0/

Supplementary Information accompanies the paper on the Leukemia website (http://www.nature.com/leu) 